\title{
SISTEMA DE INFORMACIÓN Y SATISFACCIÓN DEL USUARIO EN LA DIRECCIÓN DE PROYECCIÓN SOCIAL DE LA FAE - UNCP
}

\section{INFORMATION SYSTEM AND USER SATISFACTION IN THE DIRECTION OF SOCIAL PROJECTION FAE - UNCP}

\author{
Nieto Julcamanyan, Raúl Moisés *, Soto Salazar, Noé Chedorlaomer **, \\ Flores De La Cruz Iscela (1), Arana Olivera Sheyla Izamar (2), Soto Galvez Karen Nohelly (3)
}

\section{RESUMEN}

Uno de los ejes centrales de la Universidad es la de servir a la sociedad, aplicando los conocimientos obtenidos en las aulas, esta es una actividad muy importante de los profesionales, estudiantes y docentes. La Dirección de Proyección Social de la Facultad de Administración de Empresas de la Universidad Nacional del Centro del Perú es la que se encarga de administrar estas actividades, planificando organizando, dirigiendo estas tareas, teniendo gran parte de la responsabilidad a su cargo. Esta administración actualmente se observa en estado muy deficiente, con respuesta lenta e inoportuna a los cambios constantes en la realidad, debido a herramientas muy tradicionales y en desuso que perjudica a los usuarios del servicio prestado por la Dirección de Proyección de Social, generándoles descontento e insatisfacción. Por este motivo el objetivo principal de esta tesis es determinar cómo se relaciona un Sistema de Información con el nivel de satisfacción de los usuarios de los servicios de la Dirección de Proyección Social de la Facultad de Administración de Empresas de la Universidad Nacional del Centro del Perú. Si contar con un Sistema de Información en la Dirección de Proyección de Social de la Facultad de Administración de Empresas de la Universidad Nacional del Centro del Perú influye directamente en el nivel de satisfacción de los usuarios de los servicios. Finalmente después del desarrollo de la investigación se concluyó en que si existe una relación directa entre el Sistema de Información y la Satisfacción del Usuario de los servicios de la Dirección de Proyección Social de la Facultad de Administración de Empresas de la Universidad Nacional del Centro del Perú. Por tanto se hace necesario contar con un Sistema de Información en esta dirección, como en otras de similar categoría, orientando los resultados de la gestión a satisfacer las expectativas de los usuarios. El trabajo de investigación es necesario e indispensable en el contexto actual ya que nos ha permitido identificar adecuadamente el método de Formulación de Programas con la Metodología del Marco Lógico en el Perú, como una estrategia de asignación optima y de calidad de los escasos recursos con que cuenta la economía nacional, asimismo nos ha permitido analizar y obtener conclusiones sobre el Marco Lógico.

Palabras Clave: Programas, Sistema de Información, Proyección Social, Satisfacción del Usuario, Evaluación.

\section{ABSTRACT}

One of the cornerstones of the University is to serve society, applying knowledge gained in the classroom, this is a very important activity of professionals, students and teachers. The Directorate of Social Projection of the Faculty of Business Administration, National University of Central Peru is in charge of managing these activities, planning organizing, directing these tasks, taking much of the responsibility for their care. This administration is currently observed in very poor condition, with slow and inconvenient response to constantly changing reality due to very traditional and obsolete tools which penalizes users of the service provided by the Department of Social Projection, generating discontent and dissatisfaction. Therefore the main objective of this thesis is to determine

\footnotetext{
* Universidad Nacional del Centro del Perú - Facultad de Administración de Empresas - IIFAE, E-mail: raul1407_1@ hotmail.com

** Universidad Nacional del Centro del Perú - Oficina de Programación de Inversiones - OGPLAN Mail: nsotos@hotmail.com (1,2 y 3$)$ Colaboradores Externos
} 
the degree of relationship of an information system with the level of satisfaction of users of the Social Screening of the Faculty of Business Administration, National University of Center Peru. The hypothesis is "The degree of relationship between Information System and User Satisfaction Social Outreach office of the Faculty of Business Administration, National University of Central Peru High". So we proceeded to gather information to observe the relationship between the two variables, as the study population having users Office Social Projection. Finally after the development of the investigation concluded that there is a high degree of relationship between Information and User Satisfaction Services and the Social Screening of the Faculty of Business Administration from the Universidad National of Center Peru. In conclusion, it is necessary to have an information system in this direction, as in other similar category, guiding the management performance to meet the expectations of users.

Keywords: Programs, Budget, System of Public Investment, Evaluation, Efficiency, Efficacy and Efectivity of the Expense, Logical Framework.

\section{INTRODUCCIÓN}

En un escenario mundial globalizado y competitivo, las organizaciones, se empeñan en ser cada vez mejores, recurriendo a todos los medios disponibles para lograr sus objetivos. Se dice que una organización es buena o mala, dependiendo de la calidad de sus recursos humanos y de la gestión y resultados que éstos logran. Es por ello, el presente trabajo de investigación titulado "Sistema de Información y Satisfacción del Usuario en la Dirección de Proyección Social de la Facultad de Administración de Empresas de la Universidad Nacional del Centro del Perú", nos va permitir saber si las diferentes gestiones que realizan los usuarios en la Oficina de Proyección Social, los dejan satisfechos, teniendo como resultado la obtención de lo solicitado en el tiempo y la calidad esperada.

El Objetivo General del Proyecto de Investigación establecido es, Determinar de qué manera se relaciona un Sistema de Información en la Satisfacción del Usuario en la Dirección de Proyección Social de la Facultad de Administración de Empresas de la Universidad Nacional del Centro del Perú.

Del mismo modo, el marco de referencia: fundamentos teóricos, que ha orientado el desarrollo del presente trabajo de investigación, constituye la base fundamental en la revisión bibliográfica utilizada. Finalmente, se presentarán las propuestas pertinentes conducentes a la mejora de la satisfacción de los usuarios del servicio prestado por la Oficina de Proyección Social en la Facultad de Administración de Empresas de la Universidad Nacional del Centro del Perú que también podría aplicarse por similitud a otros centros de estudios superiores del país. El presente trabajo de Investigación busca ayudar a entender y manejar los principios básicos del enfoque del Marco Lógico y su forma de aplicación para el diseño y posterior evaluación de proyectos y programas. Algunas de las razones para realizar el trabajo de investigación se basan en la renovada inquietud por contar con un instrumento, a la vez versátil y sumamente potente, para apoyar a la llamada Gestión para Resultados, en los programas y proyectos del sector público.

\section{MATERIAL Y MÉTODOS}

El Lugar de ejecución de la investigación es la UNCP - Dirección de Proyección Social de la Facultad de Administración de la FAE - UNCP. Para la realización del Proyecto de Investigación ha sido necesaria la revisión y utilización de diversos sistemas administrativos de la Administración Pública. En general documentos de gestión institucional de la UNCP.

Materiales, Ios materiales y equipos utilizados durante el proceso de Investigación, fueron:

Bibliografía, textos recientes, propios, de bibliotecas particulares y de la Universidad. - Uso de Internet y correo electrónico, propios. - Útiles de materiales de escritorio de propiedad de los ejecutores. - Una Computadora e impresora con software adecuado, propios. - Movilidad para la recolección de datos, alquilada. - Equipos humanos expertos para aplicación de entrevistas, terceros. 


\section{Población y Muestra}

Para el análisis del estudio se ha identificado a la población usuaria de la Dirección de Proyección Social de la FAE - UNCP. Asimismo la muestra representativa ha estado constituida principalmente por la FAE UNCP, donde se ha obtenido los datos necesarios para la Investigación. Población $=$ Muestra $=64$.

\section{Metodología}

La metodología utilizada ha sido el método funcionalista, como método general del proceso de Investigación y dentro de ella también se ha utilizado los métodos descriptivo, inductivo, deductivo; tomando como realidad práctica la administración pública, caso de la Dirección de Proyección Social - FAE - UNCP. Con la finalidad de determinar en la praxis la problemática real y proponer un Sistema de Información Gerencial para lograr satisfacción del usuario.

\section{Variables Perturbadoras}

Las conocidas variables perturbadoras, las que se han constituido en las variables de análisis y estudio. Son:

Sistema de Información - VI

Satisfacción del Usuario - VD - Dimensiones:

1. Rendimiento percibido

2. Expectativas,

3. Nivel de Satisfacción.

Técnicas y Procedimientos de Recolección de Datos Entre las técnicas y procedimientos de recolección de datos básicos se usó:

- Observación y análisis.

- Entrevistas Directas

- Acceso a la información vía Internet.

- Correo Electrónico

- Observación Directa de análisis documental y bibliográfico mediante técnicas de fichaje.

\section{Técnicas de Procesamiento y Análisis de Datos}

- El análisis estadístico, mediante los cuadros y gráficos estadísticos, para interpretar los resultados del trabajo de campo.

- Análisis de los datos a partir de la recopilación de los datos obtenidos en el proceso de investigación. Mediante la metodología de análisis - síntesis.
- Contrastación de la Hipótesis, con la evidencia empírica para lograr resultados valederos.

\section{RESULTADOS}

Durante el Proceso de Investigación se ha abordado, varios aspectos referidos a realizar un estudio sistémico de la Dirección de Proyección Social de la FAE UNCP, para lograr satisfacción del usuario. Para lograr los objetivos de la presente investigación se aplicó un pre y post estudio de las variables involucradas.

\section{ANTES DE LA IMPLANTACION DEL SISTEMA DE INFORMACION COMPUTARIZADO}

\section{A. Variable Dependiente: Satisfacción del Usuario}

La variable dependiente "Satisfacción de los Usuarios" de la Oficina de Proyección Social de la Facultad de Administración de Empresas de la Universidad Nacional del Centro del Perú, se aplicó una pre y post encuesta, para poder observar su variabilidad en relación a la variable independiente "Sistema de Información" que se evaluó mediante una pre y post observación de la situación del servicio de Proyección Social antes y después de la implantación del SI

Satisfacción del usuario - rendimiento percibido:

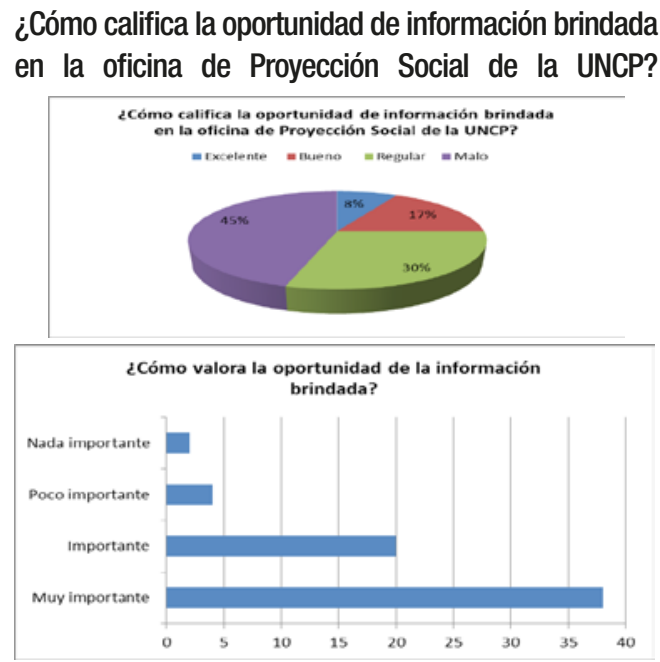

Fuente: Cuestionario de encuesta 
Se observa que en la oficina de Proyección Social no se cuenta con un Sistema de Información o con un programa básico de mantenimiento de equipos de cómputo.

Asimismo no se cuenta con un registro sistematizado de los Proyectos de los usuarios, lo cual no permite una atención oportuna a sus solicitudes y reclamos, esto también dificulta la administración adecuada de la Dirección de Proyección Social.

\section{DESPUES DE LA IMPLANTACION DEL SISTEMA DE INFORMACION COMPUTARIZADO}

\section{A. VARIABLE INDEPENDIENTE: SISTEMA DE INFORMACIÓN}

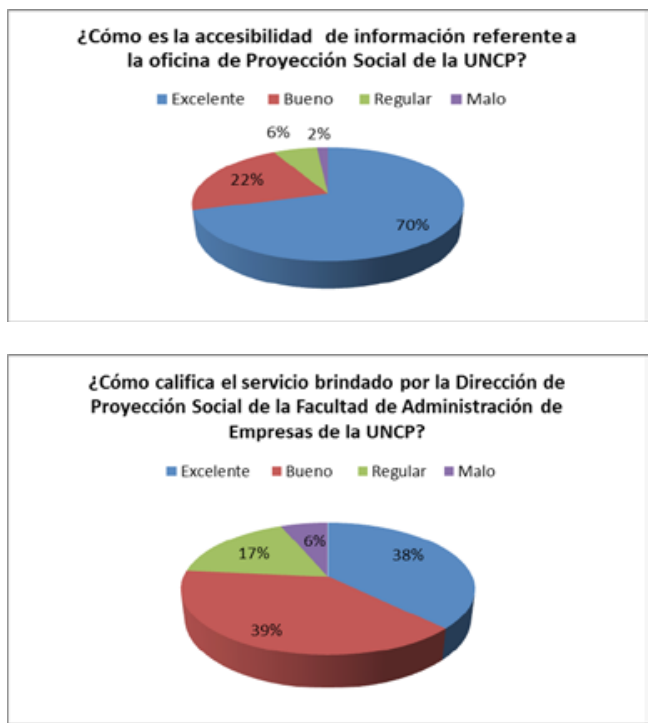

\section{DISCUSIÓN}

EXPECTATIVAS DEL USUARIO: DESPUES DE LA IMPLANTACION DEL SISTEMA DE INFORMACION RENDIMIENTO PERCIBIDO

\begin{tabular}{|l|c|c|c|c|}
\hline \multicolumn{1}{|c|}{ ITEMS } & \multicolumn{4}{|c|}{ Valoración } \\
\hline & Excelente & Bueno & Regular & Malo \\
\hline & 1 & 2 & 3 & 4 \\
\hline $\begin{array}{l}\text { ¿Cómo califica la oportunidad de in- } \\
\text { formación brindada en la oficina de } \\
\text { Proyección Social de la UNCP? }\end{array}$ & & $x$ & & \\
\hline $\begin{array}{l}\text { ¿Qué opina del nivel de precisión de la } \\
\text { información brindada en la oficina de } \\
\text { Proyección Social de la UNCP? }\end{array}$ & $X$ & & & \\
\hline $\begin{array}{l}\text { ¿Cómo califica la absolución de re- } \\
\text { clamos en la Dirección de Proyección } \\
\text { Social? }\end{array}$ & & $x$ & & \\
\hline
\end{tabular}

\begin{tabular}{|l|l|l|l|l|}
\hline $\begin{array}{l}\text { ¿Cómo es la accesibilidad de infor- } \\
\text { mación referente a la oficina de Pro- } \\
\text { yección Social de la UNCP? }\end{array}$ & X & & & \\
\hline $\begin{array}{l}\text { ¿Cómo considera el nivel de agilidad } \\
\text { en la facilitación de información por } \\
\text { la oficina de Proyección Social de la } \\
\text { UNCP? }\end{array}$ & $X$ & & & \\
\hline $\begin{array}{l}\text { ¿Cómo califica el servicio brindado } \\
\text { por la Dirección de Proyección Social } \\
\text { de la Facultad de Administración de } \\
\text { Empresas de la UNCP? }\end{array}$ & & $X$ & & \\
\hline RENDIMIENTO PERCIBID0 (MAL0) & & X.53 & & \\
\hline
\end{tabular}

Fuente: Cuestionario de encuesta

El Coeficiente de determinación nos indica que existe una relación directa entre ambas variables y por el valor 0.999485587, el cual se acerca considerablemente a la unidad, la relación es casi perfecta, es decir el nivel de satisfacción de los usuarios se ve muy influenciada por la utilización de un Sistema de Información en la Oficina de Proyección Social de la Facultad de Administración de Empresas de la UNCP.

El nivel de correlación entre las variables, nos dá a conocer el "Coeficiente de Determinación", el cual es R² $=0.999485587$, que ha sido calculado en Excel, según los índices de la Tabla № 18.

\begin{tabular}{lcc}
\hline & $\begin{array}{c}\text { Sistema de infor- } \\
\text { mación (Variable } \\
\text { independiente) }\end{array}$ & $\begin{array}{c}\text { Nivel de Satisfac- } \\
\text { ción del usuario } \\
\text { (Variable depen- } \\
\text { diente) }\end{array}$ \\
\hline $\begin{array}{l}\text { Sistema de información } \\
\text { (Variable independiente) }\end{array}$ & 1 & \\
\hline $\begin{array}{l}\text { Nivel de Satisfacción del } \\
\text { usuario (Variable depen- } \\
\text { diente) }\end{array}$ & 0.99974276 & \\
\hline
\end{tabular}

COEFICIENTE DE DETERMINACIÓN R^2

0.999485587

\section{CONCLUSIONES}

- Según los resultados de la investigación existe un alto grado de relación entre el nivel de satisfacción de los usuarios de los servicios de Proyección Social en la Facultad de Administración de Empresas de la UNCP y el Sistema de Información (Determinado por el coeficiente de correlación equivalente a 0.9997).

- Un Sistema de Información mejora el nivel de rendimiento percibido por los usuarios, por ello la visión del usuario es determinante para el éxito o fracaso de un Sistema de Información (El promedio obtenido acerca del rendimiento percibido por los usuarios 
es de 8.53, lo cual indica que con un Sistema de Información los usuarios de los servicios de Proyección Social de la Facultad de Administración de Empresas de la UNCP, califican el rendimiento como Bueno).

- Un Sistema de Información satisface las expectativas de los usuarios. Con un sistema de información se cubre las expectativas, reduciendo esa brecha entre los percibido y lo esperado (El puntaje promedio obtenido acerca de las expectativas de los usuarios es de 2.89, lo cual indica que los usuarios de la oficina de Proyección Social de la Facultad de Administración de Empresas de la UNCP, tienen altas expectativas referente al servicio brindado en la misma y un Sistema de Información cubre estas expectativas).

- Existe un grado de satisfacción positiva del usuario con la utilización de un Sistema de Información. Esta evaluación debido a que un Sistema de Información permite mejorar las deficiencias en la calidad de información y la oportunidad de respuesta a sus solicitudes. (Con un Sistema de Información se observa que el usuario está satisfecho, por los beneficios que brinda el mismo, obteniendo un puntaje resultante de 5.64).

\section{REFERENCIAS BIBLIOGRÁFICAS}

- Arellano Vílchez Mario Alfonso, Tesis: "Uso de la Tecnología de Información y Comunicación para mejorar la Calidad de Servicio de taxi en la Empresa Taxi Line 212121 en la ciudad de Huancayo" - 2011.

- Bustamante Millalonco, Jessica, Tesis: Análisis de la percepción sobre la satisfacción de los usuarios en el Hospital Regional de Valdivia- Chile - 2008.

- Hernández Sampiere, Fernández Collado Carlos, Baptista Lucio Pilar, Roberto, Fernández: “Metodología de la Investigación, Colombia, Mc. Graw Hill. 2006.

- Méndez, C.: Metodología - Guía para Elaborar Diseños de Investigación en Ciencias Económicas, Contables y Administrativas. Mc Graw Hill, Segunda Edición. Colombia. 2000.

- Stoner, J., Freeman C. y Gilbert, D. Administración. Prentice Hall, México. 2006.

- Obrien, J y Marakas, G. Sistemas de Información Gerencial. 7ma Edición. Mc Graw Hill. 2006.

- INEl: Metodología para la elaboración de una Plan de Sistemas de Información. INEI, Perú. 2002.
- Rander, B. y Heizer J. Principios de Administración de Operaciones. Prentice Hall, México. 2006.

- PC MAGAZINE. Revista de Computadoras Vol. 12 No. 5 Televisa S.A., México. 2008.

- Werther, W.B. y Davis, K.: Administración de Personal y Recursos Humanos. México: McGraw Hill. 1996.

- Miguel, A. Pequeña Empresa. Palmir, Lima. Perú. 2002.

- Kotler y Armstrong: Fundamentos de Marketing, 6ta Edición, Págs. 10, 11. 2005.

- Sawmong, Sudaporn y Ogenyi Omar: La tienda de fidelización de los consumidores al por menor del Reino Unido. The Journal of American Academy of Business Cambridge. 05 de septiembre, Págs.503509. 2004.

- Zeithaml, Valerie A. y Mary Jo Bitner: Marketing de Servicios. Un enfoque de integración del cliente a la empresa. Segunda edición. McGraw Hill. México Págs.94-107. 2002.

- Kristensen, Kai y Anne Martensen: Medición de la satisfacción del cliente: una dimensión clave de rendimiento empresarial. Revista Internacional de Gestión de Rendimiento de Negocio, vol. 2. Págs.157-170. 2000.

- Stanton, et. al.: Fundamentos de Marketing. McGraw Hill. (Décimo cuarta edición español) McGraw Hill. Págs.490 -515. 2007.

- Laudon Kenneth C. y Laudon Jane P.: "Sistemas de Información Gerencial”. Editorial Pearson Educación, Octava Edición, México. Págs.8-92. 2004.

- Cohen, Daniel. y Asin Enrique: "Sistemas de Información para los Negocios", McGraw-Hill / Interamericana S.A., Tercera Ed., México. Págs.3-22. .2006.

- James A. O'Brien y George M. Marakas. "Sistemas de Información Gerencial”. Editorial McGraw - Hill/ Interamericana S.A., Séptima Edición, México, 2006.

Referencia en Línea:

$\checkmark$ TERESA GARCÍA LÓPEZ http://www.uv.mx/iiesca/personal/garcia.html\#publicaciones

$\checkmark$ JOSÉ THOMAS MILANO. http://www.monografias.com 